\title{
Diabetes mellitus tipo 2 em idade pediátrica
}

Cláudia Raínho, ${ }^{1}$ Ana Margarida Adão, ${ }^{2}$ Inês Teles, ${ }^{1}$ Margarida Neto ${ }^{3}$

\section{RESUMO}

Introdução: A crescente incidência de diabetes mellitus tipo 2 (DM2) em idade pediátrica é uma realidade. O rastreio recomenda-se perante fatores de risco, sendo comuns ao adulto os critérios diagnósticos. Nesta idade o tratamento visa o controlo glicémico e de fatores de risco associados, sem comprometer o desenvolvimento, e também a prevenção de comorbilidades e complicações. Este caso clínico pretende destacar a abordagem holística precoce e o envolvimento familiar na abordagem da DM2 neste grupo etário.

Descrição do caso: Adolescente, sexo masculino, 12 anos, hispânico, com antecedentes pessoais de obesidade, alimentação desequilibrada e história familiar de obesidade e DM2, recorreu à consulta por glicemia capilar ocasional elevada, referindo polidipsia. Apresentava glicemia em jejum 160mg/dl e HbA1C 9,6\%. Foi referenciado à especialidade de endocrinologia e, após estudo, iniciou alterações de estilo de vida e terapêutica farmacológica com metformina. Atualmente, após quatro anos, apresenta-se normoponderal, com $\mathrm{HbA} 1 \mathrm{C}<6 \%$, apenas sob terapêutica não farmacológica.

Comentário: A abordagem desta patologia em idades jovens exige um trabalho conjunto de autoridades políticas, rede escolar e cuidados de saúde para uma promoção de estilos de vida saudáveis e combate ativo à obesidade infantil, procurando envolver e responsabilizar os cidadãos. Os profissionais de saúde devem estabelecer precocemente o diagnóstico e terapêutica para evitar complicações, sendo essenciais as mudanças de estilo de vida e o envolvimento familiar.

Palavras-chave: Diabetes mellitus tipo 2; Crianças; Adolescentes.

\section{INTRODUÇÃO}

A diabetes mellitus tipo 2 (DM2) é uma doença crónica associada a elevadas taxas de morbilidade e mortalidade, cuja incidência tem aumentado mundialmente. ${ }^{1}$ Recentemente, o número de novos casos em crianças e adolescentes tem aumentado significativamente, relacionado com o incremento da obesidade infantil e sedentarismo. ${ }^{2-6} \mathrm{~A}$ sua abordagem constitui um desafio nesta faixa etária, nomeadamente no diagnóstico diferencial com a diabetes mellitus tipo 1 (DM1), uma condição mais frequente nas crianças e jovens. ${ }^{2}$ A maioria dos casos em idade pediátrica surge na adolescência, relacionada com a insulino-resistência típica da puberdade. ${ }^{3,7}$ Neste contexto, é recomendado o rastreio nas crianças e adolescentes na presença de fatores de risco, sendo comuns ao adulto os critérios diagnósticos (Tabela 1).,2,7

1. Médica Especialista. USF Arte Nova, ACeS Baixo Vouga. Aveiro, Portugal. 2. Médica Especialista. USF Senhora de Vagos, ACeS Baixo Vouga. Aveiro, Portugal. 3. Médica Especialista. USF Leme, ACeS Baixo Vouga. Aveiro, Portugal.
Os principais objetivos na abordagem da criança ou do adolescente com DM2 incluem o controlo da glicemia, o controlo do peso e dos fatores de risco associados (e.g., a hipertensão arterial [HTA] e a dislipidemia), a promoção de alterações do estilo de vida e a prevenção de comorbilidades e complicações agudas e crónicas, sem comprometer o ritmo normal de crescimento e desenvolvimento. ${ }^{2,6,8}$

O previsível aumento de novos casos de DM2 em crianças e adolescentes ao longo dos próximos anos justifica a necessidade de melhorar a sua prevenção, diagnóstico e tratamento precoces.

Este caso clínico destaca o valor da abordagem holística, do recurso a estratégias adequadas ao adolescente e do envolvimento familiar no diagnóstico e tratamento da DM2 em idade pediátrica.

\section{DESCRIÇÃO DO CASO}

Em 2013 recorreu à consulta um adolescente de 12 anos por glicemia capilar ocasional elevada. Tratava-se de um adolescente do sexo masculino, hispânico, 
TABELA 1. Rastreio e critérios de diagnóstico para DM2 na criança e adolescente

Rastreio de DM2 em idade pediátrica²

IMC superior ao percentil 85 para a idade e sexo + pelo menos, dois dos seguintes:

- História de DM2 em familiar de primeiro ou segundo grau

- Raça/etnia de risco (americano-indiana, áfrico-americana, hispânica, asiática/Ilhas do Pacífico)

- Sinais de insulinorresistência ou comorbilidades associadas (acantose nigricans, hipertensão arterial, dislipidemia, síndroma do ovário poliquístico)

- História materna de diabetes ou de diabetes gestacional na gestação

Idade de início: 10 anos de idade ou início da puberdade, se a puberdade ocorrer em idade mais precoce

Frequência: trianual

Critérios de diagnóstico em idade pediátrica²

- Glicemia em jejum $\geq 126 \mathrm{mg} / \mathrm{dL}$

- Glicemia ocasional $\geq 200 \mathrm{mg} / \mathrm{dL}$ com sintomas

- PTGO* $\geq 200 \mathrm{mg} / \mathrm{dL}$ após as 2 horas

- $\mathrm{Hb} \mathrm{A} 1 \mathrm{C} \geq 6,5 \%$

*Em jovens com idade igual ou inferior a 15 anos, a PTGO é realizada com 1,75g de glicose/quilograma de peso até $75 \mathrm{~g}$ e $1 \mathrm{dL}$ de água por cada $25 \mathrm{~g}$ de glicose ${ }^{11}$

Na ausência de sintomas de hiperglicemia, os resultados devem ser confirmados repetindo o teste.

residente na Venezuela até aos nove anos de idade. Gravidez e parto sem intercorrências, nomeadamente diabetes gestacional, com somatometria adequada à idade gestacional. Antecedentes pessoais de obesidade e de hábitos alimentares desequilibrados, com consumo excessivo de hidratos de carbono simples, nomeadamente refrigerantes, e pobre em fruta e legumes. $\mathrm{Ne}$ gava outros antecedentes relevantes. Enquanto residiu na Venezuela não teve seguimento em consultas de vigilância, não sendo portador de nenhuma informação médica prévia. Antecedentes familiares de obesidade, DM2, HTA e doença cardiovascular precoce. Pertence a uma família nuclear (Figura 1), altamente funcional, que se encontra na fase $\mathrm{V}$ do ciclo de vida de Duvall. No círculo de Thrower o adolescente representou-se equidistante dos restantes elementos da família nuclear, referindo estar satisfeito com a sua representação (Figura 2).

O adolescente recorreu à consulta por queixas de polidipsia com um mês de evolução, motivo pelo qual a mãe realizou uma pesquisa de glicemia capilar apresentando um valor de 300mg/dL. Negava poliúria, polifagia, perda ponderal, astenia ou visão turva. Ao exa- me objetivo apresentava fenótipo obeso, sem acantose ou estrias violáceas, proporcionado, sem outras alterações de relevo. Normotenso (tensão arterial de $110 / 60$ inferior ao percentil 90 para a altura, sexo e idade). Relativamente aos dados antropométricos, peso de $80 \mathrm{~kg}$; altura de $170 \mathrm{~cm}$ (>P97), IMC de $27,7 \mathrm{Kg} / \mathrm{m}^{2}$ (muito superior ao P97). Realizada pesquisa de glicemia capilar ocasional na consulta, apresentando $323 \mathrm{mg} / \mathrm{dL}$. Foi explicado ao adolescente a importância de modificar os estilos de vida com cuidados alimentares e incremento da atividade física e pedidas análises que revelaram uma hemoglobina glicada (HbAlc) de 9,6\%, glicemia em jejum 160mg/dl e ficha lipídica normal. Após contacto telefónico com o serviço de endocrinologia do hospital de referência foi marcada consulta urgente.

Na consulta hospitalar, cinco dias depois, foi pedido novo estudo analítico e consulta de nutrição, tendo iniciado metformina 500mg, com aumento progressivo até 1000mg bidiário. Após cinco meses de modificação de estilos de vida e terapêutica farmacológica tinha uma HbAlc de 5,9\% e uma redução ligeira do IMC. Os autoanticorpos (Ac Anti-GAD, Ac Anti-Ilhéus de 


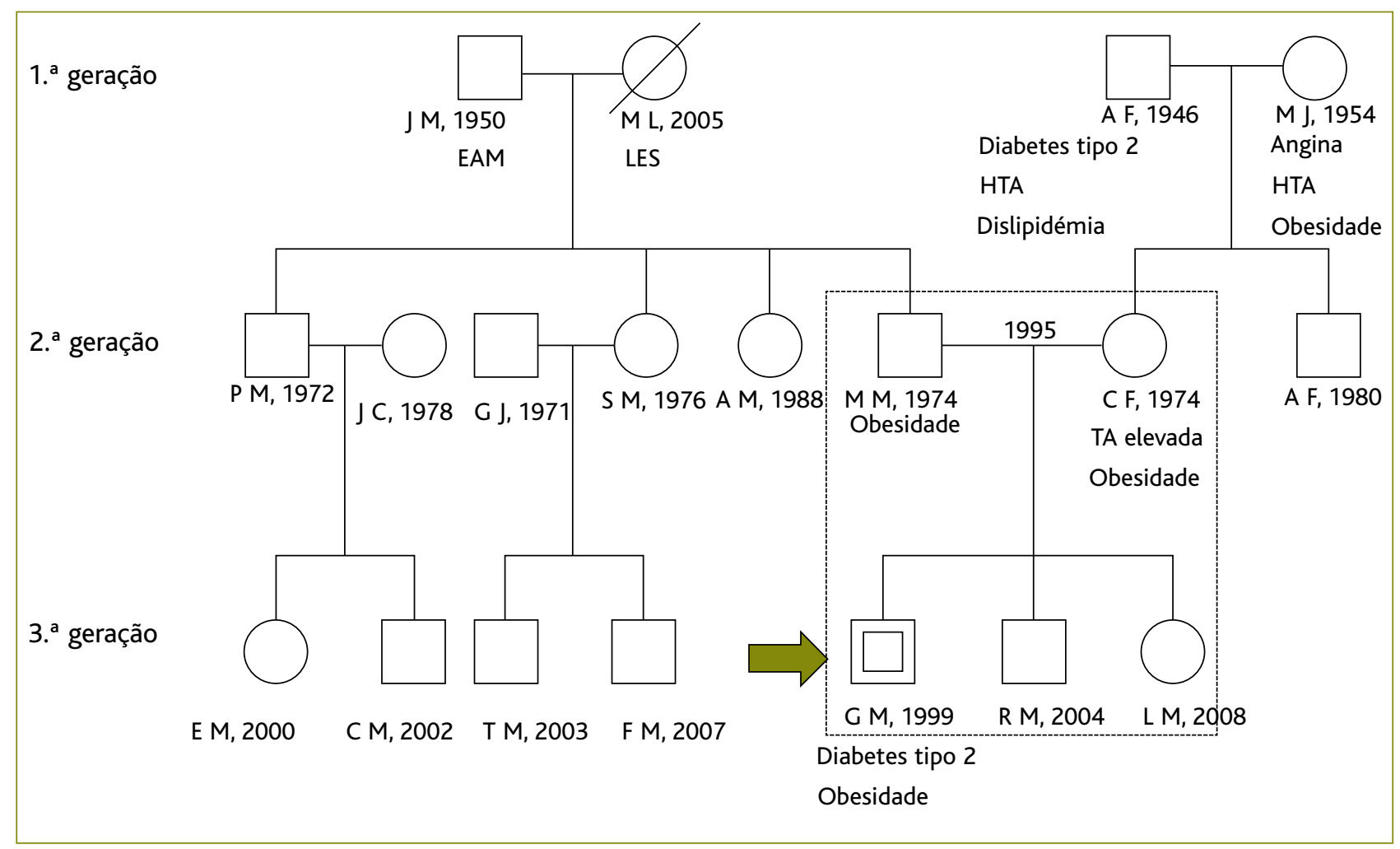

Figura 1. Genograma familiar (maio de 2013).

Legenda: EAM = Enfarte agudo do miocárdio; LES = Lúpus eritematoso sistémico; HTA = Hipertensão arterial.

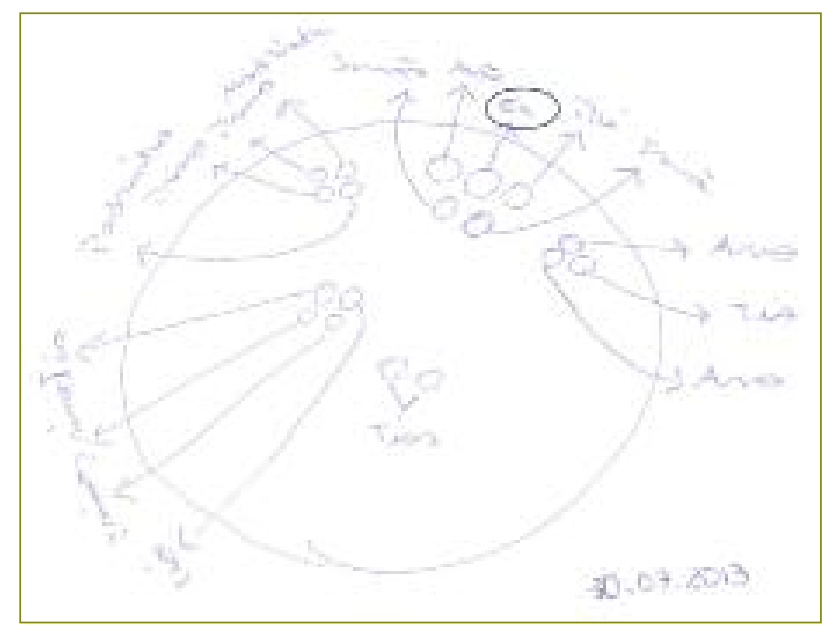

Figura 2. Círculo familiar de Thrower.

Langerhans [AICA] e Ac. Anti-Insulina), a pesquisa de microalbuminúria e o rastreio de retinopatia diabética foram negativos. Manteve seguimento em consultas de endocrinologia e nutrição, tendo aderido a modifica- ções de estilo de vida rigorosas, com restrição de alimentos ricos em hidratos de carbono simples, aumento do consumo de frutas e vegetais e aumento da atividade física, nomeadamente com natação e caminhadas. Verificou-se redução progressiva do IMC, encontrando-se atualmente normoponderal (Figura 3). A HbAlc manteve-se entre 5 e $6 \%$, pelo que foi suspensa metformina após três anos de terapêutica. Cerca de seis meses após suspensão farmacológica os valores analíticos mantiveram-se estáveis, mantendo apenas medidas não farmacológicas. A nível familiar verificou-se uma adesão às mudanças de estilo de vida iniciadas pelo adolescente, do que tem resultado em redução do IMC dos progenitores, nomeadamente da mãe com decréscimo de 36 para $28 \mathrm{~kg} / \mathrm{m}^{2}$.

\section{COMENTÁRIO}

ADM2 e as comorbilidades associadas são fatores de risco para doenças micro e macrovasculares futuras. $\mathrm{O}$ seu início em idade pediátrica é particularmente 


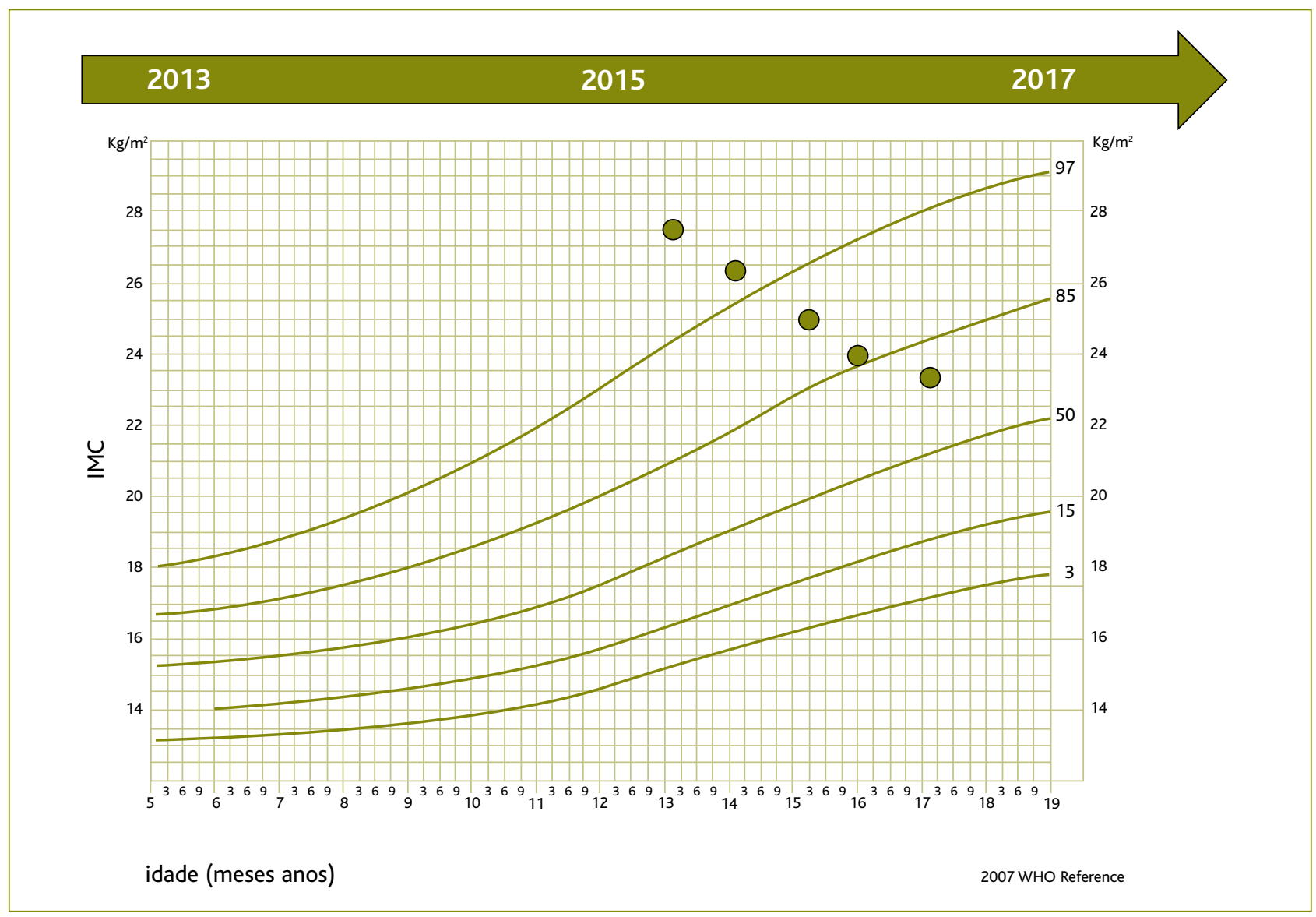

Figura 3. Evolução do IMC do adolescente entre 2013 e 2017.

preocupante, uma vez que o tempo de evolução, a par do nível de controlo da doença, é um dos principais preditores de aparecimento de complicações. ${ }^{6}$

Segundo a Associação Americana de Diabetes, devem ser rastreadas as crianças e adolescentes com risco elevado para desenvolver DM2 (Tabela 1), recorrendo a glicemia em jejum, prova de tolerância oral à glicose e eventualmente HbAlc, embora a validade desta última no diagnóstico seja questionada em alguns estudos. ${ }^{2,7,9}$

Os critérios de diagnóstico são semelhantes aos do adulto, exigindo-se, na ausência de sintomas, repetição para confirmação do diagnóstico (Tabela 1). ${ }^{2}$ Os sintomas sugestivos incluem polidipsia, poliúria, polifagia, perda ponderal, astenia, cicatrização lenta, visão turva, infeções vaginais recorrentes ou manifestações agudas, como cetoacidose diabética ou coma hiperosmolar hi- perglicémico. ${ }^{5}$ Entre as possíveis causas de hiperglicemia, o principal diagnóstico diferencial é a DM1. ${ }^{5} \mathrm{O}$ início mais insidioso, o fenótipo de insulinorresistência (obesidade, acantose nigricans) e a ausência de autoanticorpos apoiam a hipótese de DM2. ${ }^{5}$

Outras situações menos comuns a considerar em contextos de hiperglicemia incluem fibrose quística, outras patologias endócrinas (síndroma de Cushing, feocromocitoma), hiperandrogenismo ovárico e iatrogenia (corticoides, antiretrovirais e quimioterapia). ${ }^{5}$

As atuais recomendações terapêuticas resultam fundamentalmente da experiência acumulada no tratamento de adultos, sendo as mudanças de estilo de vida e o envolvimento familiar pilares centrais na abordagem da DM2 em todas as idades. Nas crianças e adolescentes têm papel primordial o investimento numa alimentação saudável e equilibrada e atividade física 
regular, restringindo alimentos ricos em hidratos de carbono simples e «tempo de ecrã»., ${ }^{5,7}$ As terapêuticas aprovadas para uso pediátrico incluem insulina e metformina, sendo recomendada terapêutica inicial com insulina em caso de cetose ou cetoacidose, dúvida diagnóstica, glicemia $\geq 250 \mathrm{mg} / \mathrm{dl}$ ou HbAlc $>9 \%$. Nos restantes casos deve ser iniciada metformina. ${ }^{2,7-8}$ A metformina deve ser iniciada na dose de 500-1000mg diários durante uma a duas semanas, devendo ser titulada a dose semanalmente durante três a quatro semanas, dependendo da tolerância do utente, até uma dosagem máxima de 1000mg bidiária. Os efeitos secundários digestivos (dor abdominal, diarreia, náusea) podem ocorrer, mas podem ser minimizados com a titulação gradual da dosagem ao longo de três a quatro semanas, com a ingestão simultânea de alimentos ou através do uso de formulações de libertação prolongada. O risco de acidose láctica é extremamente reduzido.

Este adolescente tinha indicação para iniciar insulina pelas glicemias elevadas, mas foi iniciada terapêutica com metfomina por indicação da endocrinologista.

O ajuste terapêutico progressivo baseia-se na $\mathrm{HbAlc}$, sendo recomendável o valor alvo $<7 \%$. $^{7}$

Após o diagnóstico e durante o seguimento a longo prazo, a International Society for Pediatric and Adolescent Diabetes recomenda monitorização da HbAlc trimestralmente e da pressão arterial em todas as consultas, pesquisa de microalbuminúria, retinopatia, dislipidemia e esteatose hepática não alcoólica à data do diagnóstico e anualmente, bem como o rastreio regular de alterações pubertárias e menstruais e apneia obstrutiva do sono. ${ }^{7}$

Neste caso clínico destaca-se o controlo glicémico através de terapêutica não farmacológica, após fase inicial de terapêutica com metformina e o envolvimento familiar através da educação de toda a família, permitindo aumentar a adesão do doente com ganhos em saúde dos vários elementos da família.

Pela relação privilegiada com o utente, contacto continuado no tempo e conhecimento e acesso ao contexto familiar, o médico de família tem uma posição de destaque no seguimento destas patologias, primando pela otimização e individualização dos recursos empregues em cada indivíduo. Neste caso clínico, o médi- co de família foi o primeiro profissional envolvido no diagnóstico e primeira abordagem terapêutica, iniciando a explicação da fisiopatologia da doença adaptada ao conhecimento e compreensão do adolescente e família e valorizando as mudanças de estilo de vida fundamentais ao sucesso da terapêutica. Numa abordagem inicial de diabetes ou suspeita de diabetes em idade pediátrica deve ser avaliada a cetonémia ou cetonúria, pois, se positiva, indica tratamento urgente e o adolescente deve ser referenciado ao serviço de urgência pelo risco de cetoacidose. ${ }^{7} \mathrm{O}$ adolescente e a família revelaram precocemente interesse nessas mudanças, que foram sugeridas de acordo com as preferências destes: natação como exercício físico preferido do adolescente e caminhadas como atividade física mais fácil de adaptar ao seu quotidiano e envolvendo todo o agregado familiar. Relativamente aos cuidados alimentares foi solicitado ao adolescente que elaborasse um diário alimentar para uma segunda consulta, onde foram discutidas algumas alterações a realizar de acordo com o diário apresentado. Ao longo das consultas realizadas foram avaliados os ganhos e propostas mudanças cada vez mais exigentes e adaptadas aos ganhos e motivação do adolescente. Inicialmente o adolescente foi avaliado com uma periocidade mensal, que foi alargada à medida que as alterações de estilo de vida foram rigorosamente incluídas, culminando no excelente controlo metabólico rapidamente alcançado. A entrevista motivacional é fundamental para a mudança de estilos de vida, sendo particularmente desafiante na adolescência. A empatia, a disponibilidade, o tempo dedicado em cada consulta, que ultrapassou largamente o tempo previsto de consulta, as decisões conjuntas e o reforço positivo de cada ganho alcançado foram fundamentais para a mudança verificada. Este caso ilustra a dificuldade sentida pelos profissionais de saúde relativamente ao tempo padrão para consultas particularmente destinadas à prevenção primária, que se revela muito exigente, mas prioritária. Por outro lado, os desafios relacionados com a acessibilidade (nomeadamente, a dimensão da lista de utentes) condicionam a capacidade do médico de família programar um acompanhamento tão frequente quanto o desejável, sobretudo nas fases iniciais.

Conforme ilustrado neste relato, a articulação com os cuidados hospitalares é particularmente 
importante para a realização de exames complementares de diagnóstico, fundamentais para o diagnóstico e indisponíveis para o médico de família, para orientação terapêutica e integração em equipa multidisciplinar, incluindo serviços de acesso mais restrito nos cuidados de saúde primários, como a consulta de nutrição e psicologia.

O previsível aumento de novos casos de DM2 em crianças e adolescentes nos próximos anos justifica a necessidade de apostar na sua prevenção, diagnóstico e tratamento precoces. Apesar de inegável a carga genética associada à DM2, a obesidade é o fator de risco modificável com maior contributo e um combate ativo a esta problemática terá certamente impacto positivo na incidência da DM2 em idades jovens. O estabelecimento de medidas políticas promotoras de estilos de vida saudáveis, o envolvimento dos cuidados de saúde primários, nomeadamente em conjunto com a rede escolar, e a definição de estratégias de responsabilização dos cidadãos com promoção do autocuidado permitirá, a médio e longo prazo, obter ganhos em saúde, nomeadamente no controlo das doenças cardiovasculares, com uma melhor gestão dos recursos disponíveis e necessários.

Por fim, atendendo à possibilidade de prevenção e atraso do aparecimento desta patologia e ao benefício do seu controlo para evitar complicações associadas, é fundamental que os profissionais de saúde estejam sensibilizados para a DM2 em idade pediátrica, de forma a estabelecer precocemente o diagnóstico e a terapêutica adequada.

\section{REFERÊNCIAS BIBLIOGRÁFICAS}

1. Observatório da Diabetes. Diabetes: factos e números - O ano de 2014 (relatório anual do Observatório Nacional da Diabetes). Lisboa: Socie- dade Portuguesa de Diabetologia; 2015. ISBN 978-989-96663-2-0

2. American Diabetes Association. Standards of medical care in diabetes - 2016: summary of revisions. Diabetes Care. 2016;39(Suppl 1):S4-5.

3. Temneanu OR, Trandafir LM, Purcarea MR. Type 2 diabetes mellitus in children and adolescents: a relatively new clinical problem within pediatric practice. J Med Life. 2016;9(3):235-9.

4. Lee PH. Association between adolescents' physical activity and sedentary behaviors with change in BMI and risk of type 2 diabetes. PLoS One. 2014;9(10):e110732.

5. Tieh P, Dreimane D. Type 2 diabetes mellitus in children and adolescents. Indian J Pediatr. 2014;81(2):165-9.

6. Kao KT, Sabin MA. Type 2 diabetes mellitus in children and adolescents. Aust Fam Physician. 2016;45(6):401-6.

7. Phelan H, Lange K, Cengiz E, Gallego P, Majaliwa E, Pelicand J, et al. ISPAD clinical practice consensus guidelines 2018: diabetes education in children and adolescents. Pediatr Diabetes. 2018;19(Suppl 27):75-83.

8. Copeland KC, Silverstein J, Moore KR, Prazar GE, Raymer T, Shiffman $\mathrm{RN}$, et al. Management of newly diagnosed type 2 diabetes mellitus (T2DM) in children and adolescents. Pediatrics. 2013;131(2):364-82.

9. Galhardo J, Shield J. The role of haemoglobin A1c in screening obese children and adolescents for glucose intolerance and type 2 diabetes. Acta Med Port. 2015;28(3):307-15.

10. National Institute for Health and Care Excellence. Diabetes (type 1 and type 2) in children and young people: diagnosis and management [homepage]. NICE; 2015 [updated 2020 Dec 16]. Available from: https:// www.nice.org.uk/guidance/ng18

11. Direção-Geral da Saúde. Processo assistencial integrado da diabetes mellitus tipo 2: informação n. ${ }^{\circ}$ 001/2013, de 19/02/2013. Lisboa: DGS; 2013.

\section{CONFLITO DE INTERESSES}

Os autores declaram não possuir quaisquer conflitos de interesse.

\section{ENDEREÇO PARA CORRESPONDÊNCIA}

Cláudia Raínho

E-mail: claudiacc18@hotmail.com

https://orcid.org/0000-0002-3448-8900

Recebido em 25-01-2018

Aceite para publicação em 25-02-2021 


\section{ABSTRACT}

\section{TYPE 2 DIABETES MELLITUS IN PEDIATRIC AGE}

Introduction: Type 2 diabetes mellitus (DM2) increasing incidence in the pediatric population is a reality. Screening is recommended only when risk factors are present, and the diagnostic criteria match the adults' guidelines. The aim of the treatment is glycemic and risk factors control without compromising the child or adolescent development and prevention of comorbidities and complications. This case report intends to emphasize the early holistic approach and family involvement in the DM2 approach in adolescence.

Case description: An obese 12-years old Hispanic male teenager, with a family history of obesity and DM2, presented to a medical appointment with elevated capillary blood glucose and polydipsia. Analytically, had fasting glycemia $160 \mathrm{mg} / \mathrm{dl}$ and HbA1C 9.6\%. Monitored by Endocrinology, engaged a strategy of lifestyle changes and metformin. Currently, after four years, has a normal body mass index and HBA $1 \mathrm{c}<6 \%$ only with non-pharmacological therapy.

Comment: DM2 approach demands political figures, school networks, and health care services working together to promote healthy lifestyles and an effective fight against obesity. To avoid future complications, health professionals must provide an early diagnosis and medical therapy with a particular focus on lifestyle changes and family involvement.

Keywords: Diabetes mellitus, type 2; Child; Adolescent. 